\title{
AUTOMATIC TONAL HARMONIZATION FOR MULTI-SPECTRAL MOSAICS
}

\author{
Pouya Dehghani Tafti* \\ Biomedical Imaging Group, \\ École Polytechnique Fédérale de Lausanne (EPFL), \\ Lausanne, Switzerland
}

\author{
Xiaolin Wu
Dept of Electrical and Computer Engineering, McMaster University, \\ Hamilton, Ontario, Canada
}

\begin{abstract}
When producing a mosaic of multiple multi-spectral images one needs to harmonize the colours so that the tone transition is smooth from one image to the other. Given two images $\operatorname{Im}^{a}$ and $\mathrm{Im}^{b}$, a transform $T$ is sought to map $\operatorname{Im}^{b}$ to an image that is harmonious in multi-spectral appearance to $\mathrm{Im}^{a}$. We give the above problem of tonal harmonization an analytical framework, in which both ideal and practical solutions of the problem are studied. Using a physically motivated image formation model, we prove that a perfect tonal harmonizing operator cannot in general be found, but that whenever such an operator exists it is linear. In the latter case, finding the optimal harmonizing transformation can be cast as a linear programme (LP), which is a type of problem that can be efficiently solved using known techniques. Finally, strong empirical evidence is provided for the efficacy of the proposed solution.
\end{abstract}

Index Terms - multi-spectral imaging, image mosaics, tonal harmonization, colour balancing, linear programming.

\section{INTRODUCTION}

This research is motivated by applications that deal with multispectral remote sensing images; yet the development and results hereafter are equally valid for other types of multi-spectral images (e.g., colour photographs in consumer electronics). Aerial photographs of the earth surface are typically acquired under varying illumination conditions that depend on factors such as the time of the day, season, and various atmospheric phenomena. Many applications require the composition of such photographs into an extended image mosaic that covers vast areas, impossible to capture with the desired level of detail in a single satellite image. In these applications, harmony in the colour or multi-spectral tone of adjacent blocks can be important, both for visual continuity e.g. in mapping applications, and for the purpose of identifying materials based on colour or multi-spectral measurements.

While several commercial packages for remote sensing imagery provide tonal adjustment functionality to different degrees, possibly due to trade secrets, appearance of the subject in research literature has been limited. The standard approach uses histogram matching [1]. Several histogram methods are reviewed in [2]. Another approach is suggested in [3], which is based on minimizing the difference between colour-balanced pixel values separately in individual channels, using a geometric kernel (see also [4, 5]). These approaches are typically not physically motivated. In addition, some of them are computationally expensive, and/or do not make use of inter-band information. The latter factor, as will be seen, limits the

${ }^{*}$ This work was done while the first author was with McMaster University, Canada. effectiveness of the approach when addressing imaging under varying illumination.

In contrast, the formulation we present in this paper is based on a mathematical model for the image formation process, which allows us to study tonal harmonization in a rigorous mathematical framework. Tonal harmonization, as we show in $\S 3$, is an ill-posed problem. In particular, it is proven in $\S 3$ that in general, it is not possible to find any operator (be it linear or non-linear) that can relate an arbitrary image taken under illumination $b$ to one taken under illumination $a$ without requiring additional information, which is typically not available. However, as we also show in $\S 3$, for a particular subset of reflectance functions for which such an operator can be found, it is always linear.

It should be noted here that the use of linear colour transformations has previously been suggested in the context of colour constancy [6]. There, the justification follows from the experimental observation that spectral reflectance functions and spectral distributions of illumination sources can be well approximated by finitedimensional linear expansions. In comparison, our formulation in $\S 3$ is derived from a model for the physical process of image formation. Additionally, it is sometimes suggested that a diagonal linear transform, i.e. one that treats each channel separately, is sufficient. We show examples to the contrary. It becomes clear that a full (i.e. not diagonal) linear transform that uses all spectral bands in adjusting each measurement has significant advantages.

After having established that tonal harmonization can be effectively achieved using a linear transformation, we proceed in $\S 4$ to formulate an optimization problem for finding the parameters of this transformation. In particular, we show that the problem can be posed as a case of linear programming (LP) which can then be efficiently solved using known numerical techniques. Herein lies another point of departure from more traditional tonal adjustment methods. Traditional colour harmonization approaches work on the principle of histogram matching (cf. [1], §4.5), which is typically performed by adjusting the mean, standard deviation, and range of histograms [2]. In contrast, we calculate the harmonizing transform using corresponding pixel values directly, instead of basing our calculations on agglomerate data such as histograms. Our model and approach are verified by experimental results, some examples of which we provide in this paper.

The organization of the remainder of the paper is as follows. We describe the mathematical model for the image formation process in $\S 2$. Next, in $\S 3$, we discuss the formulation of the harmonizing transformation, and provide justification for the use of a linear operator. In $\S 4$ we discuss how, given two overlapping images, the parameters of the transformation can be optimally computed. We conclude the paper in $\S 5$ with a discussion of experimental results and a few final remarks. 


\section{IMAGE FORMATION MODEL}

Light reflection from the earth surface is modelled by a spectral reflectance function (for an in depth discussion see for instance [7]). This process is explained below, and motivates the image acquisition model

We associate with the surface at spatial coordinates $(u, v)$ a nonnegative and bounded reflectance function $f_{u, v}(\lambda)$ ( $\lambda$ denotes the wavelength), and assume that when this location is illuminated by a light source with spectral distribution $I(\lambda)$, the spectral distribution of the reflected light, as observed at the acquisition device, is given by $I(\lambda) f_{u, v}(\lambda)$. (The subscript of $f_{u, v}$ may be dropped for convenience; its implicit dependence on position should be understood.)

The acquisition device is equipped with sensors of $n$ different types, each measuring a different spectral sub-band. These $n$ sensors are identified by their spectral response functions $\sigma_{j}(\lambda), j=$ $1, \ldots, n$. This allows us to describe the $j$ th-band sample of $f$ by defining a scalar-valued linear operator (i.e. a linear functional) $\Sigma_{j}$ : $\mathbf{L}^{2}(S) \rightarrow \mathbb{R}:$

$$
\Sigma_{j} f:=\int_{S} \sigma_{j}(\lambda) I(\lambda) f(\lambda) \mathrm{d} \lambda
$$

In the above definition $S$ is the spectral range of interest. The source of natural illumination in our application is always sunlight, and we can safely assume that it has no zero components over $S$; i.e., $I(\lambda)>0$ over $S$. In addition $S$ is assumed to be bounded, in accordance with the range of wavelengths observable by our device.

We define the vector-valued linear operator $\Sigma: \mathbf{L}^{2}(S) \rightarrow \mathbb{R}^{n}$ that has $\Sigma_{j}$ 's as its rows:

$$
\Sigma f:=\left(\Sigma_{j} f\right)_{1 \leq j \leq n} .
$$

Let $\mathcal{R}$ be a region, i.e. a set of positions $(u, v)$; and let $f_{\mathcal{R}}$ be defined as

$$
f_{\mathcal{R}}:=\left\{f_{u, v}\right\}_{(u, v) \in \mathcal{R}} .
$$

An image 'Im' covering the area $\mathcal{R}$ then corresponds to an indexed set of measurements $\left\{\Sigma f_{u, v}\right\}_{(u, v) \in \mathcal{R}}=: \Sigma f_{\mathcal{R}}$.

We denote different illumination conditions by lower case letters $(a, b, e t c$.), and label the corresponding symbols accordingly (writing, e.g., $\operatorname{Im}^{a}, \operatorname{Im}^{b}, I^{a}, I^{b}$, and $\Sigma^{a}, \Sigma^{b}$ ).

It is assumed that $\sigma_{j}$ 's are chosen such that the operator $\Sigma$ is of full rank. This is valid in practice (as otherwise the same information could have been collected using fewer sensors). ${ }^{1}$ The operator $\Sigma$, which maps an infinite-dimensional space $\left(\mathbf{L}^{2}:=\mathbf{L}^{2}(S)\right)$ into a finite-dimensional one $\left(\mathbb{R}^{n}\right)$, should therefore be onto. It is clearly not one-to-one, and is therefore not invertible. But if we exclude the null-space of $\Sigma$ from $\mathbf{L}^{2}$, that is, if we select a subspace $V$ of $\mathbf{L}^{2}$ such that

$$
\mathbf{L}^{2}=V \oplus \operatorname{null} \Sigma,
$$

$\left.\Sigma\right|_{V}$ (the restriction of $\Sigma$ to $V$ ) will be both one-to-one and onto, hence invertible. This allows a unique decomposition of an arbitrary $f \in \mathbf{L}^{2}(S)$ into the sum of two components $f_{V} \in V$ and $f_{N} \in$ null $\Sigma$. Moreover, $\Sigma$ can be factorized as

$$
\Sigma=\left.\Sigma\right|_{V} P_{V}
$$

where $P_{V}$ is the (oblique) projection operator onto $V$ along null $\Sigma$. Trivially then $f_{V}=P_{V} f$ and $f_{N}=\left(i d-P_{V}\right) f=: P_{N} f$.

\footnotetext{
${ }^{1}$ This assumption is easy to verify in practical settings. The support of the spectral response of any sensor should be at least partially disjoint from the union of all others. Consequently, the rows of $\Sigma$ (the operators $\Sigma_{j}, 1 \leq j \leq$ $n$ defined in (1)) cannot be linearly dependent.
}

\section{TONAL HARMONIZATION}

Let us now consider the problem of multi-spectral harmonization for two images acquired under different lighting conditions $a$ and $b$ (captured by operators $\Sigma^{a}$ and $\Sigma^{b}$ ). Ideally, we would like to find an operator $T$ that maps an image taken under lighting condition $b$ to what would have been captured under lighting condition $a$. In other words, we seek a map $T$ that makes the diagram

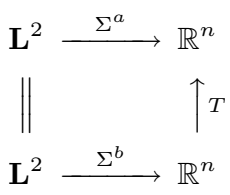

commute $;^{2}$ i.e., one that satisfies

$$
T \Sigma^{b}=\Sigma^{a} .
$$

However, as can be seen from the following lemma, this is impossible if null $\Sigma^{b} \not \subset$ null $\Sigma^{a}$.

Lemma 1 Let $A$ and $B$ be two linear maps from $X$ into $Y$. If a (not necessarily linear) map $T: Y \rightarrow Y$ exists that satisfies

$$
T B=A,
$$

we should necessarily have null $B \subset$ null $A$.

Proof (by reductio ad absurdum): Assume that the lemma is false and null $B \not \subset$ null $A$. Let $f \in X, g \in$ null $B-$ null $A \neq \varnothing$. Then

$$
A(f+g)=T B(f+g)=T(B f+B g)=T B f=A f,
$$

and hence

$$
0=A(f+g)-A f=A(f+g-f)=A g,
$$

i.e. $g \in$ null $A$, which contradicts the false premise.

For later reference we also record the next lemma.

Lemma 2 Given linear operators $A$ and $B$, if there exists a map $T$ such that $T B=A, T$ should be linear over the range of $B$.

Proof: For $f, g \in \operatorname{ran} B$, let $f_{0} \in B^{-1} f$ and $g_{0} \in B^{-1} g$ (where $B^{-1} f$ is the pre-image of $f$ ). Then:

$$
\begin{aligned}
T(\alpha f+g) & =T\left(\alpha B f_{0}+B g_{0}\right)=T B\left(\alpha f_{0}+g_{0}\right) \\
& =A\left(\alpha f_{0}+g_{0}\right)=\alpha A f_{0}+A g_{0} \\
& =\alpha T B f_{0}+T B g_{0}=\alpha T f+T g
\end{aligned}
$$

that is, $T$ is linear over $\operatorname{ran} B$.

As the requirement of Lemma 1 that

$$
\text { null } \Sigma^{b} \subset \text { null } \Sigma^{a}
$$

is not satisfied in general, we cannot find a $T$ that makes diagram (4) commute for all $f$. However, for a restricted subspace of $\mathbf{L}^{2}$ that excludes null $\Sigma^{b}-$ null $\Sigma^{a}$, (5) can be satisfied for the restriction of $\Sigma^{a}$ and $\Sigma^{b}$ to this subspace. Furthermore, we learn from Lemma 2 that if it is indeed possible to make (4) commute, this can be achieved by a linear operator $T$.

\footnotetext{
${ }^{2} \mathrm{~A}$ diagram such as the above is said to commute (or to be commutative) if following any two paths between any two particular sets defines the same map. Two parallel lines in such a diagram indicate equality.
} 
In fact, if $V$ is an $n$-dimensional subspace of $\mathbf{L}^{2}$ that satisfies (3) for $\Sigma=\Sigma^{b}$, a linear operator $T$ exists that works for all

$$
f \in V \oplus\left(\text { null } \Sigma^{a} \cap \text { null } \Sigma^{b}\right),
$$

in the sense that it makes the following diagram commute:

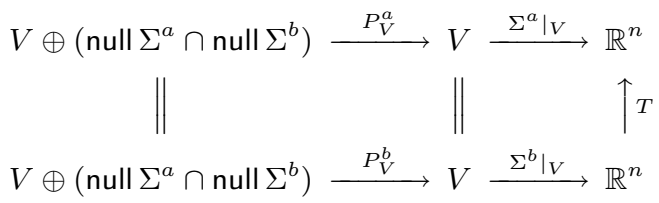

Indeed, the above diagram is commutative when $T=\left.\left.\Sigma^{a}\right|_{V} \Sigma^{b}\right|_{V} ^{-1}$ (over $\left.\operatorname{ran} \Sigma^{b}\right|_{V}$ ).

\section{COMPUTATION OF THE HARMONIZING TRANSFORMATION}

The tonal harmonization problem can be described as follows. Given two sets of observations (two images)

$$
\operatorname{Im}^{a}:=\Sigma^{a} f_{\mathcal{A}}:=\left\{\Sigma^{a} f_{u, v}\right\}_{(u, v) \in \mathcal{A}}
$$

and

$$
\operatorname{Im}^{b}:=\Sigma^{b} f_{\mathcal{B}}:=\left\{\Sigma^{b} f_{u, v}\right\}_{(u, v) \in \mathcal{B}},
$$

of intersecting regions $\mathcal{A}$ and $\mathcal{B}$, taken under illumination conditions $a$ and $b$ respectively, wanted is a transformation $T$ that, when applied to the second image of the intersection of $\mathcal{A}$ and $\mathcal{B}$ (i.e. $\Sigma^{b} f_{\mathcal{A} \cap \mathcal{B}}$ ), generates an image that is close to the first observation of the same region (i.e. $\Sigma^{a} f_{\mathcal{A} \cap \mathcal{B}}$ ), in an optimal sense. Once found, we could then apply the same transformation to the entirety of $\operatorname{Im}^{b}$ to get an image that is harmonious to $\operatorname{Im}^{a}$.

Notice that the measurements, i.e $\Sigma^{a} f_{\mathcal{A}}$ and $\Sigma^{b} f_{\mathcal{B}}$, are all that we have access to. $f_{\mathcal{A}}$ and $f_{\mathcal{B}}$ themselves, as well as the operators $\Sigma^{a}$ and $\Sigma^{b}$, are all unknown. As was made clear in the previous section, in the case of general $f_{u, v}$, the best we can possibly hope for is to find a map $T$ that minimizes some distance between $T \Sigma^{b} f_{\mathcal{A} \cap \mathcal{B}}$ and $\Sigma^{a} f_{\mathcal{A} \cap \mathcal{B}}$, as a perfect recovery of the first image from the second is in general not possible. In addition, in the light of the discussion given in the previous section, it is appropriate to choose $T$ to be linear (corresponding to an $n \times n$ matrix).

To formulate an objective for optimization, we need a measure of distance between $\Sigma^{a} f_{\mathcal{A} \cap \mathcal{B}}$ and $T \Sigma^{b} f_{\mathcal{A} \cap \mathcal{B}}$, which we choose to be a sum of point-wise distances:

$$
\sum_{(u, v) \in \mathcal{A} \cap \mathcal{B}} \rho\left(\Sigma^{a} f_{u, v}, T \Sigma^{b} f_{u, v}\right),
$$

where $\rho$ is some distance in $\mathbb{R}^{n}$.

An optimization problem can therefore be formulated thus:

$$
\operatorname{minimize}_{T \in \mathbb{R}^{n \times n}} \sum_{(u, v) \in \mathcal{A} \cap \mathcal{B}} \rho\left(\Sigma^{a} f_{u, v}, T \Sigma^{b} f_{u, v}\right) .
$$

In particular, if $\rho$ is chosen to be the $\ell_{1}$ norm, using standard convex optimization techniques it is not difficult to show that the optimization problem can be decoupled across the rows of the matrix $T$, and may then be cast as $n$ independent cases of linear programming (LP), which are efficiently solvable using available software packages that implement established approaches such as simplex or interior-point methods. If even higher computational efficiency is desired, one may instead choose to use the $\ell_{2}$ norm, leading to a linear least squares problem that has a direct analytical solution.

In our ANSI C implementation, we have successfully used the freely available lp_solve library (simplex-base; see [8] for licensing information). Harmonizing images of several thousand by several thousand pixels can be done in a matter of seconds on a typical workstation.

\section{EXPERIMENTAL RESULTS AND CONCLUSIONS}

In this paper we proposed a method for the harmonization of multispectral images. The formulation of this method was based on a model of the imaging process. After proving the non-existence of a perfect harmonizing map in the general case, we showed that whenever a perfect harmonizing map can be found, it is linear. Subsequently, we focused on linear harmonizing transformations, and suggested a solution that can be efficiently computed via linear programming. In the following, we provide sample outputs and comparisons to some other available solutions. For better comparison, high-resolution versions may be requested by writing to the authors.

A mosaic of 14 aerial photographs appears in Fig. 1(a). In Figs 1(b) and 1(c), the colour-balanced outputs of two leading commercial solutions can be seen. The output of our software appears in Fig. 1(d). In all three cases, all images have been colour-matched to the one in the middle. To produce Fig. 1(d), we matched images pairwise, first harmonizing the neighbours of the central block, then proceeding with the neighbours of the neighbours and so on.

While the outputs of the commercial solutions are visually consistent to some extent, they introduce artefacts and outlier colours, which even affect the reference image. As a result, the output is different from raw satellite imagery. This also renders these approaches less suitable for colour-based remote sensing. In contrast, our solution provides an output that is close to an image of the whole area, taken under the same conditions as the reference image. (Some discontinuity in transient phenomena (such as clouds) is visible between certain blocks, due to the fact that the images were taken at different times. If desired, this issue may be addressed by blending the two images over their common intersection (which we have not done here), or more advanced model-based techniques may be used.)

Also, as can be seen in Figs 2(a)-(c), the results are better than those obtained by a diagonal linear transform.

As a final remark, while the proposed method was initially developed for harmonization of aerial photographs, due to the generality of the formulation, this approach is also applicable to other types of images. This is demonstrated in Figs 3(a)-(c), where the same method is used for balancing digital photographs. Advantages and possible limitations of this method for colour balancing of different types of images remain to be further investigated. The choice of the optimality criterion used in optimization $(\S 4)$ also needs to be further studied.

\section{REFERENCES}

[1] J. A. Richards and X. Jia, Remote Sensing Digital Image Analysis: An Introduction, Springer, 4th edition, 2006.

[2] R. A. A. Nobrega and J. A. Quintanilha, "Automatic digital image balancing," in Joint ISPRS Workshop High Resolution Mapping from Space 2001, University of Hannover, 2001, pp. 153-159, Hannover: ISPRS. 


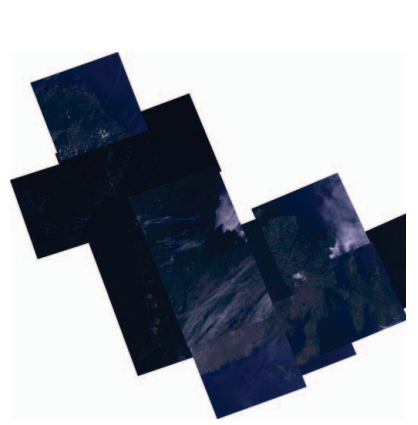

(a)

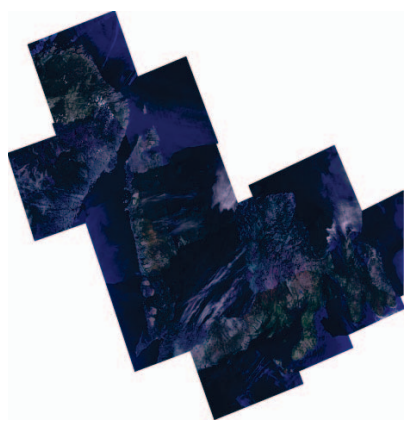

(b)

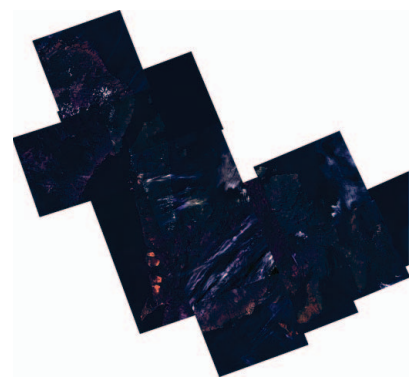

(c)

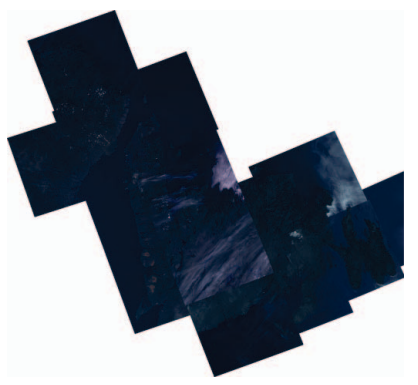

(d)

Fig. 1. Comparison of different harmonization methods: (a) original mosaic; (b), (c) colour balanced mosaics created by two leading commercial packages; (d) mosaic created using our harmonization method. In all three cases, images have been harmonized against the one in the centre. Notice that, in addition to introducing fewer artefacts, our approach is the only one that creates a mosaic that is similar to real satellite imagery. In contrast, outlier colours appear in (b) and (c). (Raw data courtesy of PCI Goematics.)

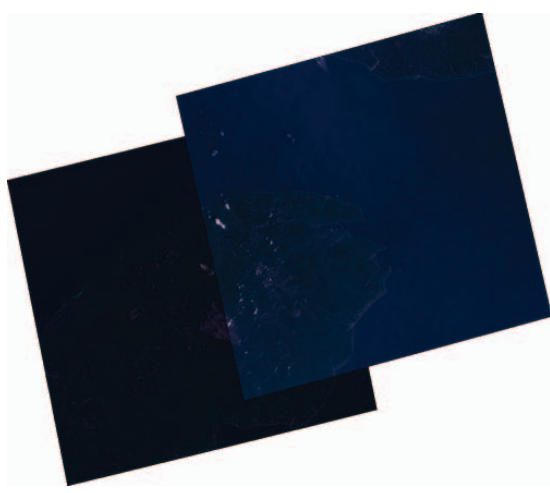

(a)

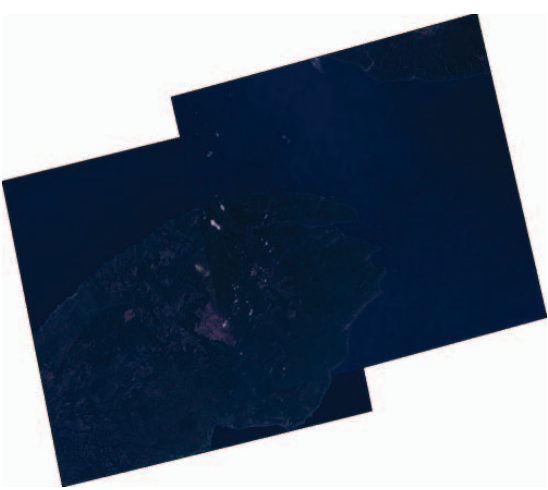

(b)

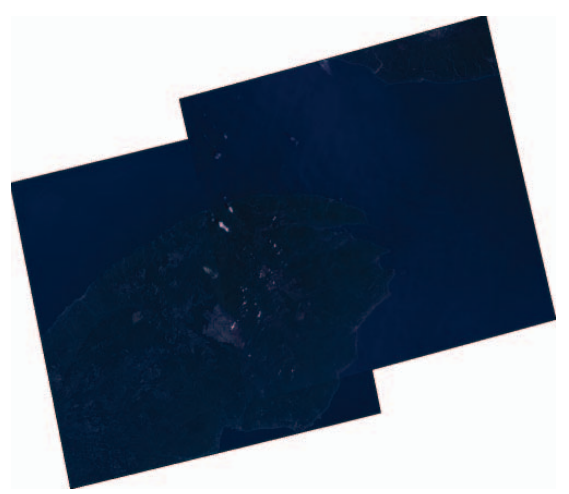

(c)

Fig. 2. Comparison of diagonal and full linear harmonizing transforms: (a) original mosaic; (b) harmonization by a median-matching linear transform; (c) mosaic created using our harmonization method. (Raw data courtesy of PCI Goematics.)

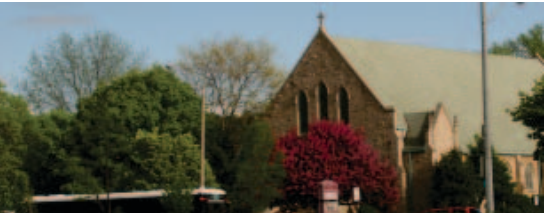

(a)

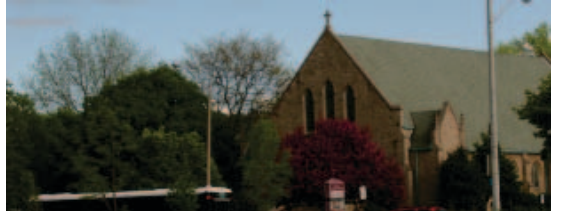

(b)

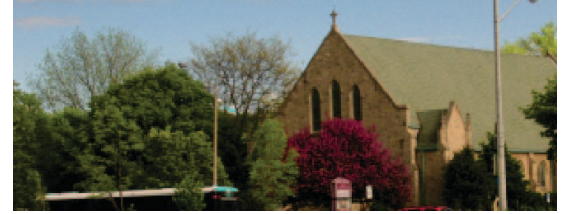

(c)

Fig. 3. Application of the proposed colour harmonization method in colour balancing: (a), (b) original images, taken with small time delay on a partly cloudy day; (c) result of harmonizing (b) against (a).

[3] X. Wu and N. A. Campbell, "A colour-balancing method and its applications," in The 12th Australasian Remote Sensing and Photogrammetry Conference, Fremantle, Spatial Science Institute, Western Australia, 18-22 Oct. 2004.

[4] C. Sun, R. Beare, V. Hilsenstein, and P. Jackway, "Mosaicing of microscope images," in DICTA '05: Proceedings of the Digital Image Computing on Techniques and Applications, Washington, DC, USA, 2005, p. 50, IEEE Computer Society.

[5] C. Sun, R. Beare, V. Hilsenstein, and P. Jackway, "Mosaicing of microscope images with global geometric and radiometric corrections," Journal of Microscopy, vol. 224, no. 2, pp. 158-165,
2006.

[6] L. T. Maloney and B. A. Wandell, "Color constancy: a method for recovering surface spectral reflectance," J. Opt. Soc. Am. A, vol. 3, no. 1, pp. 29-33, Jan. 1986.

[7] F. van der Meer and S. M. de Jong, Eds., Imaging Spectrometry: Basic Principles and Prospective Applications, Springer, 2003.

[8] "Ip_solve, a Mixed Integer Linear Programming (MILP) solver," Available online at http://lpsolve.sourceforge.net/5.5/; see the web-page for credits and licensing details. 